\title{
BMJ Open Value of early critical care transthoracic echocardiography for patients undergoing mechanical ventilation: a retrospective study
}

\author{
Hao Jiang (D) , , Wen Xu (D) , ${ }^{3}$ Wenjing Chen (D) , ${ }^{1,4}$ Lingling Pan (D) , \\ Xueshu Yu (D) ,' Yincai Ye (D) , 5 Zhendong Fang (D) ,' Xianwei Zhang (D) ,' \\ Zhiqiang Chen (D) , ${ }^{1}$ Jie Shu (D) , ${ }^{1}$ Jingye Pan (1) ${ }^{1,6}$
}

To cite: Jiang $\mathrm{H}, \mathrm{Xu} \mathrm{W}$, Chen W, et al. Value of early critical care transthoracic echocardiography for patients undergoing mechanical ventilation: a retrospective study. BMJ Open 2021;11:e048646. doi:10.1136/ bmjopen-2021-048646

- Prepublication history and additional supplemental material for this paper are available online. To view these files, please visit the journal online (http://dx.doi.org/10.1136/ bmjopen-2021-048646)

Received 03 January 2021 Accepted 07 October 2021

\section{Check for updates}

(C) Author(s) (or their employer(s)) 2021. Re-use permitted under CC BY-NC. No commercial re-use. See rights and permissions. Published by BMJ.

For numbered affiliations see end of article.

Correspondence to

Dr Jingye Pan;

wmupanjingye@126.com

\section{ABSTRACT}

Objectives To evaluate whether early intensive care transthoracic echocardiography (TTE) can improve the prognosis of patients with mechanical ventilation (MV). Design A retrospective cohort study.

Setting Patients undergoing MV for more than 48 hours, based on the Medical Information Mart for Intensive Care III (MIMIC-III) database and the eICU Collaborative Research Database (eICU-CRD), were selected.

Participants 2931 and 6236 patients were recruited from the MIMIC-III database and the elCU database, respectively.

Primary and secondary outcome measures The primary outcome was in-hospital mortality. Secondary outcomes were 30-day mortality from the date of ICU admission, days free of MV and vasopressors 30 days after ICU admission, use of vasoactive drugs, total intravenous fluid and ventilator settings during the first day of MV. Results We used propensity score matching to analyse the association between early TTE and in-hospital mortality and sensitivity analysis, including the inverse probability weighting model and covariate balancing propensity score model, to ensure the robustness of our findings. The adjusted OR showed a favourable effect between the early TTE group and in-hospital mortality (MIMIC: OR 0.78; $95 \% \mathrm{Cl} 0.65$ to $0.94, \mathrm{p}=0.01$; elCU-CRD: OR $0.76 ; 95 \% \mathrm{Cl}$ 0.67 to $0.86, p<0.01$ ). Early TTE was also associated with 30 -day mortality in the MIMIC database (OR $0.71,95 \% \mathrm{Cl}$ 0.57 to $0.88, p=0.001$ ). Furthermore, those who had early TTE had both more ventilation-free days (only in elCUCRD: 23.48 vs $24.57, p<0.01$ ) and more vasopressor-free days (MIMIC: 18.22 vs 20.64, $p=0.005$; eICU-CRD: 27.37 vs $28.59, p<0.001$ ) than the control group (TTE applied outside of the early TTE and no TTE at all).

Conclusions Early application of critical care TTE during MV is beneficial for improving in-hospital mortality. Further investigation with prospectively collected data is required to validate this relationship.

\section{INTRODUCTION}

Echocardiography, which can be conveniently performed in intensive care units (ICUs), provide more information on cardiac abnormalities, including anatomical

\section{STRENGTHS AND LIMITATIONS OF THIS STUDY}

$\Rightarrow$ The large sample size from two ICU databases increased the credibility of the research.

$\Rightarrow$ Inverse probability weighting model and covariate balancing propensity score model were used to analyse the association between early TTE and in-hospital.

$\Rightarrow$ Although a sensitivity analysis was performed to make the results more reliable, some biases are inevitable.

$\Rightarrow$ Only patients from the MICU and SICU were selected to rule out the impact of severe pre-existing heart diseases or cardiac surgery.

abnormalities and functional abnormalities, ${ }^{1}$ and quickly and accurately assess haemodynamic changes in the ICU. ${ }^{2}$ Compared with other ICU diagnostic tools, the frequency of transthoracic echocardiography (TTE) use in the ICU has increased rapidly owing to the advantages of non-invasiveness, easy availability and safety. In the span of 10 years from 1999 to 2008, the use of TTE has almost doubled, but the resulting economic and guardianship costs are also increasing. ${ }^{3}$ The research on TTE mainly focusses on the management changes caused by TTE, but the impact of these changes is unclear. ${ }^{4}$ Besides, despite the release of practice guidelines for cardiac ultrasound in 2011, many clinicians still use TTE based on their clinical experience. ${ }^{5}$ In the ICU, where information from randomised controlled trials is a relative deficiency, it is significant to understand the clinical value of a commonly used diagnostic tool.

$\mathrm{MV}$ is a common and important procedure for patients in the ICU. Although respiratory failure is the greatest problem to be solved in patients undergoing MV, haemodynamic also plays a role that cannot be ignored, ${ }^{6}$ and a recent study also showed that haemodynamic 
changes caused by the influence of airway pressure affect the prognosis of patients. ${ }^{7}$ Several studies have suggested that cardiac assessment should be included in management strategies in patients undergoing mechanical ventilation (MV). TTE can be used to assess the patient's fluid response under $\mathrm{MV}^{8}$ and is ideally suited to diagnose the weaning failure of cardiac origin. ${ }^{9}$ However, there is no strong evidence to support that TTE should be performed during MV. ${ }^{10}$ Thus, understanding the clinical value of early TTE in mechanically ventilated patients is enormously important.

Based on the above questions, our study was implemented to investigate the impact of earlier TTE performance on the outcomes of critically ill adult patients with MV.

\section{MATERIALS AND METHODS \\ Database}

This study was reported in accordance with the STrengthening the Reporting of OBservational studies in Epidemiology statement. ${ }^{11}$ We collected data from Medical Information Mart for Intensive Care (MIMIC)-III v1. $4^{12}$ and the eICU Collaborative Research Database (eICU-CRD) v2.0. ${ }^{13}$ Both are extensive, free, public databases containing hospitalisation information. MIMIC covers 61532 ICU admissions for 46476 patients at the Beth Israel Deaconess Medical Center in Boston, MA, USA. The eICU-CRD covers 200859 ICU admissions from 139226 patients at 208 US hospitals. Xueshu Yu completed the required courses for the use of the database and obtained the corresponding certificate (researcher certification number 1605699 and record id 27752 407).

\section{Study cohort}

We conducted a retrospective study of mechanically ventilated adult patients from MIMIC-III; only admissions to medical intensive care units (MICUs) or surgical intensive care units (SICUs) were included, patients in a coronary care unit and cardiac surgery recovery unit were excluded. We used only the first ICU admission data for the first hospitalisation and patients 16 years of age or older who had been continuously ventilated for at least 48 hours. Patients who had incomplete datasets were excluded. Patients who underwent echocardiography less than 24 hours before MV or within 24 hours after MV were classified as the early TTE group, and the remaining patients constituted the control group (patients who had no early TTE, including TTE applied outside of the early TTE or no TTE at all).

Data were extracted from the database using structured query language code established by Serpa Neto et al. ${ }^{14}$ The following demographic data (using data from the first 24 hours of admission) were collected: age, sex, weight, race, comorbidities (chronic obstructive pulmonary disorder (COPD), asthma, congestive heart failure (CHF), chronic kidney disease (CKD), hypertension, sepsis, acute respiratory distress syndrome (ARDS)), Sequential Organ
Failure Assessment (SOFA) score, Oxford Acute Severity of Illness Score (OASIS), vital signs (mean arterial pressure (MAP), heart rate (HR)) and laboratory values (white blood cell (WBC) count, haemoglobin ( $\mathrm{Hb})$, blood urea nitrogen $(\mathrm{BUN}), \mathrm{pH}, \mathrm{pO}_{2}, \mathrm{pCO}_{2}$ and lactate). In addition, we also collected the management data for the first day of MV (tota intravenous fluid, ventilator settings, use of dobutamine and norepinephrine).

\section{Patient and public involvement}

No patients and the public were involved in any part of this study.

\section{Outcomes}

The primary outcome of the study was in-hospital mortality. Secondary outcomes were 30-day mortality from the date of ICU admission; days free of MV and vasopressors 30 days after ICU admission, use of vasoactive drugs, total intravenous fluid and ventilator settings during the first day of MV.

\section{Statistical analysis}

In order to control the confounding factors, propensity score matching (PSM) was performed. The baseline characteristics of the original cohort were stratified by TTE. The propensity score for an individual was determined based on the covariates age, sex, weight, race, HR, COPD, asthma, CHF, CKD, hypertension, ARDS, sepsis, SOFA score, OASIS, WBC, $\mathrm{Hb}, \mathrm{pH}, \mathrm{pO}_{2}, \mathrm{pCO}_{2}$ and lactate using a standard software package (matching package) with a PSM methodology. These variables were selected due to their clinical relevance. This method consisted of ranking the MV patients in the early TTE group and the control group, then selecting the TTE patients who had the highest propensity score and finding the control group patients with the closest propensity score (maximum calliper, 0.2). Both patients were then removed from consideration for matching, and the next highest patient was selected (matched 1:1 using the nearest-neighbour algorithm).

After matching, to assess the balance between the two groups, the standardised mean differences (SMDs) between the early TTE cohort and the control cohort were calculated. SMDs eliminate not only the influence of the absolute values from a study but also the influence of the unit of measurement on the results. ${ }^{15}$ Continuous variables are shown as the means and $\mathrm{SD}$, and categorical variables are represented as the total and proportion. For continuous variables, we used a non-parametric test or the Wilcoxon rank-sum test. For the categorical variables, we used a $\chi^{2}$ test or Fisher's exact test.

Secondary outcomes were observed after matching as well. We used paired t-tests for continuous outcomes and $\chi^{2}$ tests for categorical outcomes.

We used the random forest model to impute missing data (online supplemental eFigures 1 and 2). ${ }^{16}$

\section{Sensitivity analysis}

We conducted a series of sensitivity analyses with the cohort with missing data, the cohort after imputation and 


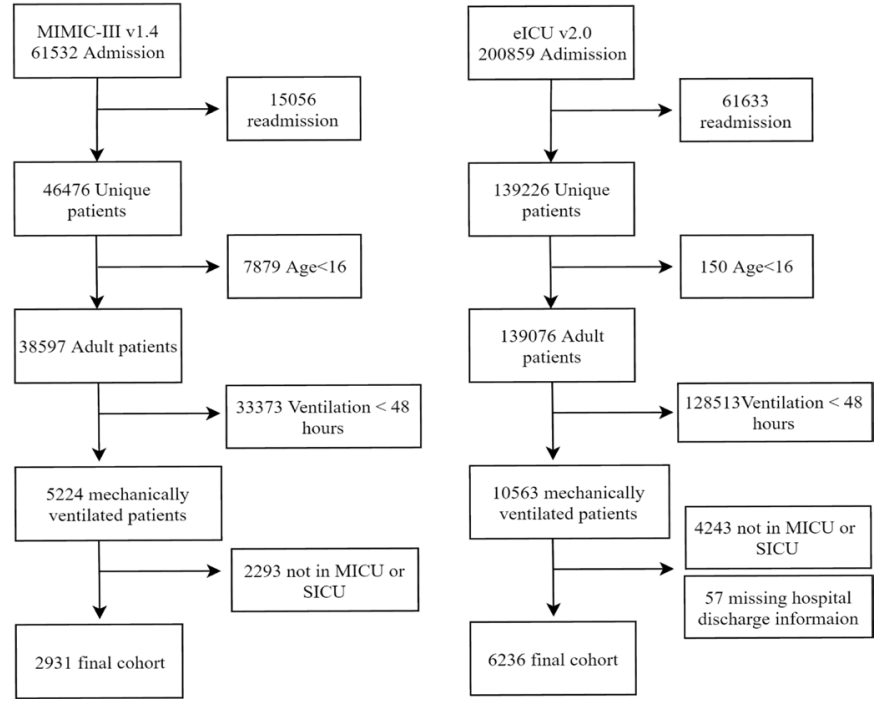

Figure 1 Flow chart illustrating the inclusion and exclusion criteria of the study. MICU, medical intensive care units; MIMIC, Medical Information Mart for Intensive Care; SICU, surgical intensive care units.

the cohort after PSM to assess the outcomes. In addition, we used multiple logistic regression, the inverse probability of treatment weight (IPTW) ${ }^{17}$ and the covariate balancing propensity score (CBPS) ${ }^{18}$ to further validate the primary outcome. To adjust for these covariates, the doubly robust estimation method ${ }^{19}$ was used to deduce the independent associations between TTE and in-hospital mortality and 30-day mortality (details about the IPTW and CBPS can be found in the online supplemental material). In addition, we divided the patients into three groups (Early TTE, late TTE (TTE time $\geq$ MV time +24 hours) and non-TTE (no TTE after MV)), and further analyses were conducted to identify predictors of early TTE using multiple logistic regression. Finally, we carried out a sensitivity analysis through multivariate logistic regression focussing on patients with ARDS and sepsis.

Statistical significance was assessed to be determined by a two-sided $\mathrm{p}<0.05$. All statistical analyses mentioned above were performed using $\mathrm{R}$ version 3.5.3.

\section{RESULTS}

After reviewing 46476 unique patients from the MIMIC-III database and excluding those with readmission, age $<16$ years, and ventilation duration $<48$ hours, 2931 patients from the MICU and SICU were enrolled (figure 1). In the eICU-CRD, of the 139226 unique patients, 6236 patients in the MICU and SICU were included after the exclusion of patients aged $<16$ years, those receiving invasive ventilation for less than 48 hours and that missing hospital discharge information (figure 1). The in-hospital mortality of MV patients was $31.53 \%$ in the MIMIC database and $28.62 \%$ in the eICU-CRD. Patients who died were older, had lower weight, higher OASIS and SOFA scores, higher lactate, and lower MAP (online supplemental eTable 1).

The original cohort baseline in the MIMIC database showed that patients who underwent TTE on the first day of MV had a more severe status in terms of the SOFA score, MAP, $\mathrm{pH}, \mathrm{pO}_{2}$ and $\mathrm{pCO}_{2}$ (table 1); however, there was no statistically significant difference between the early TTE group and the control group in the eICU-CRD (table 1).

\section{Primary outcomes}

Univariate logistic regression analysis results of in-hospital mortality are shown in online supplemental eTable 2 , and the details of multiple logistic regression are shown in online supplemental eTable 3. The clinical features of TTE during different time periods and no TTE at all are shown in online supplemental eTable 4. Then, we used PSM to standardise the differences between the early TTE group and the control group (online supplemental eTables 5 and 6). All covariates were balanced in the PSM cohort (online supplemental eFigures 3 and 4). The adjusted OR (MIMIC: OR $0.78,95 \%$ CI (0.65 to 0.94$)$, $(\mathrm{p}=0.01)$; eICU-CRD: OR $0.76,95 \% \mathrm{CI}(0.67$ to 0.86$)$, $(p<0.01))$ showed that the early use of TTE was beneficial to improve the in-hospital mortality of patients undergoing MV (figure 2).

\section{Secondary outcomes}

After PSM, early TTE was also associated with 30-day mortality in the MIMIC database (OR $0.71,95 \%$ CI $(0.57$ to 0.88 ), $\mathrm{p}=0.001$ ) (online supplemental eFigure 5). Since the eICU-CRD only contains in-hospital mortality data, we had no way to assess the association between early TTE and 30-day mortality in the eICU-CRD cohort. In addition, we found that those who had early TTE had both more ventilation-free days (but there was no statistical difference in MIMIC, $\mathrm{p}=0.051$ ) and more vasopressorfree days in 30 days than the control group (online supplemental eTable 7), which might be related to the management changes brought by early TTE, including a less amount of intravenous fluid (only in the eICU-CRD: 2156.88 (3635.99) vs. $1470(2670.69), \mathrm{p}<0.001)$, the use of vasoactive drugs and the ventilator setting parameters (only in the MIMIC database). However, this conclusion should be regarded cautiously as far as the generalisation of results is concerned.

\section{Sensitivity studies}

We performed some sensitivity analyses, as summarised in figure 2 (in-hospital mortality) and online supplemental eFigure 5 (30-day mortality). We analysed all three cohorts, including the matched cohort, the original cohort with missing data, and the cohort after imputation, and found similar results: in-hospital mortality and 30-day mortality were improved in mechanically ventilated patients undergoing early TTE. In addition, early TTE was more conducive to the prognosis of patients than late TTE and non-TTE (online supplemental eFigure 6), 
Table 1 Baseline characteristics of the original cohort

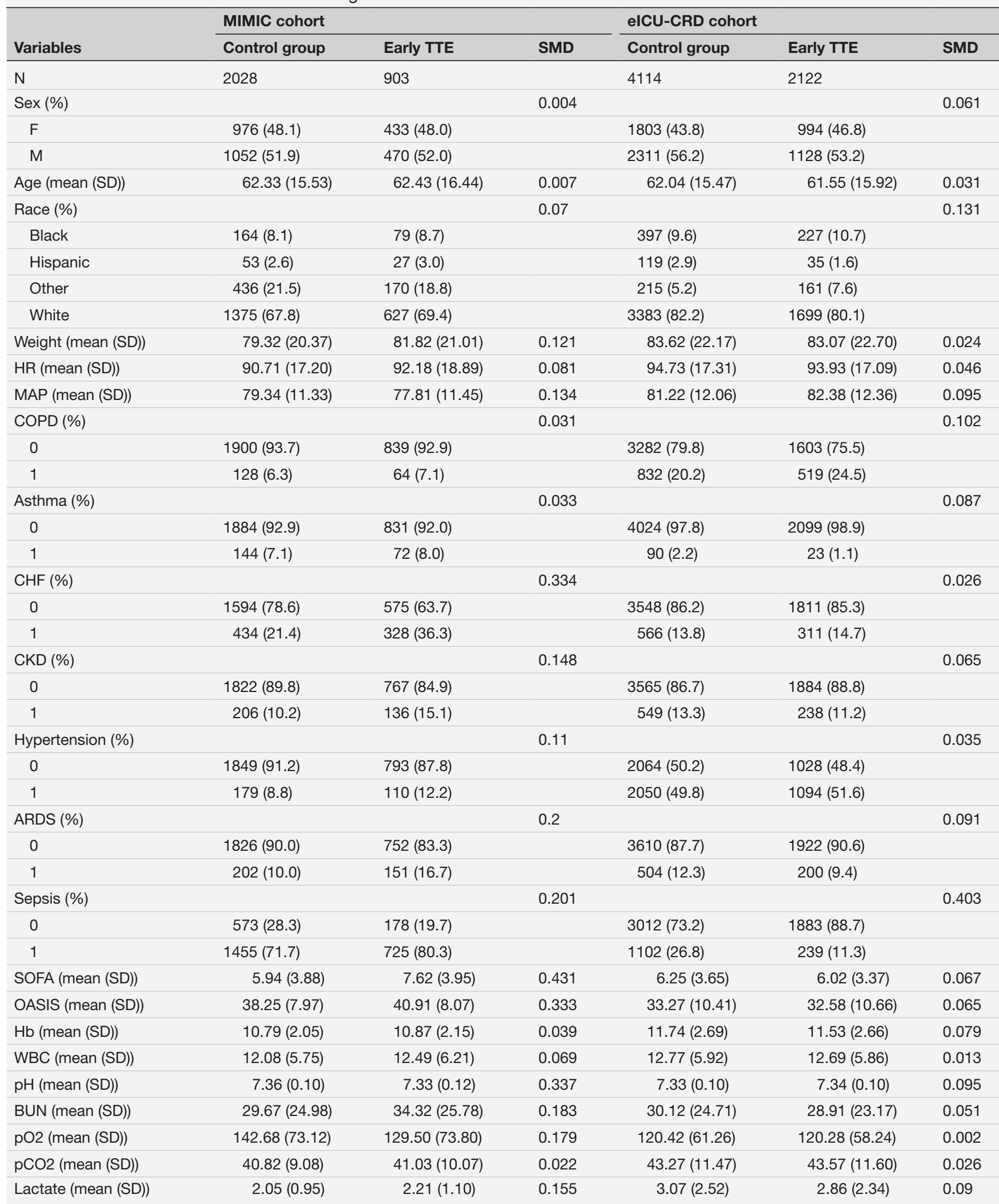

Data are reported as the mean (SD) or no./total (\%).

All data were extracted in the first 24 hours of ICU admission.

ARDS, acute respiratory distress syndrome; BUN, blood urea nitrogen; CHF, congestive heart failure; CKD, chronic kidney disease; COPD, chronic obstructive pulmonary disorder; Hb, haemoglobin; HR, heart rate; MAP, mean arterial pressure; OASIS, Oxford Acute Severity of Illness Score; SMD, standardised mean difference; SOFA, sequential organ failure assessment; WBC, white blood cell. 


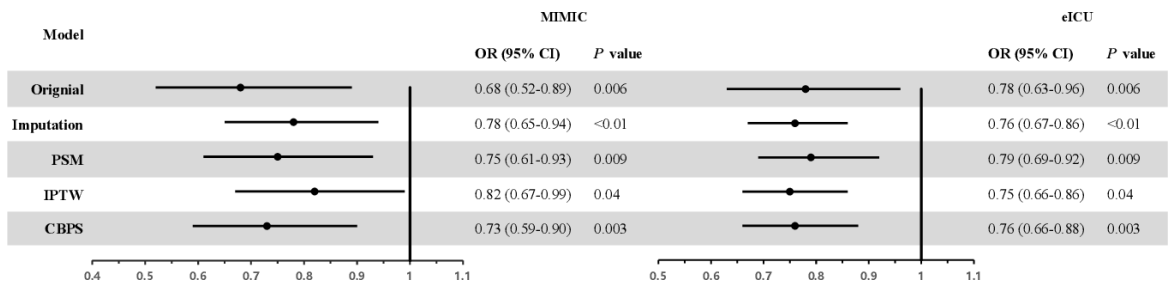

Figure 2 Primary outcomes and sensitivity analyses. The results showed that early use of transthoracic echocardiography (TTE) was beneficial in improving the in-hospital mortality of patients undergoing mechanical ventilation. sensitivity analyses were performed to determine whether the results were dependent on the method of covariate adjustment. original, the cohort with missing data; imputation, the cohort after imputation; PSM, the cohort after propensity score matching; IPTW, the cohort after inverse probability of treatment weight; CBPS, the cohort after covariate balancing propensity score.

and the subgroup analysis also showed that early TTE was beneficial in improving in-hospital mortality in the ARDS cohort (in the MIMIC sample only) and the sepsis cohort (online supplemental eTable 8). The clinical characteristics of ARDS and sepsis patients are shown in online supplemental eTable 9 .

\section{DISCUSSION}

In our study, the results provided evidence to support our hypotheses. After we adjusted for important confounding factors through PSM analysis, the results showed that early TTE was associated with a reduced risk of in-hospital mortality and 30-day mortality in these patients. The above results were verified by the data from the MIMIC III and eICU-CRD databases. We also tested several hypotheses to account for the mortality benefit. It may be that TTE caused a change in management and improved the patient's prognosis, but based on current research, we cannot be completely sure.

We consider the following possible reasons for the improvement in in-hospital mortality and 30-day mortality with echocardiography: The MV affects haemodynamic as well as the respiratory system. ${ }^{20}$ The effect of ventilation on the haemodynamic of the heart is mainly due to changes in pleural pressure ( $\mathrm{Ppl})$, and $\mathrm{Ppl}$ affects both the inflow of the right ventricle (RV) and the outflow of the left ventricle (LV).$^{21}$ While reducing the vena cava return, resulting in abnormal filling of the $\mathrm{RV}$, it will also increase the resistance of the pulmonary vascular vessels and affect the outflow function of the RV. While affecting the RV, it also affects the function of the LV. In addition, in the case of inappropriate tidal volume, blood flow is mostly blocked due to transpulmonary pressure (TP) rising to a level that exceeds pulmonary artery pressure, resulting in a substantial right ventricular afterload. ${ }^{22}$ These effects lead to acute cardiac strain and functional and even organic lesions. Intuitive assessment of cardiac function can help clinicians adjust ventilator settings to minimise the occurrence of cardiovascular dysfunction while maintaining ventilation. ${ }^{23}$

TTE is a valuable tool for monitoring haemodynamic bedside $^{24}$; it can easily provide dynamic haemodynamic parameters for assessing the type of shock that cannot be determined by clinical examination ${ }^{25}$ and information about heart-lung interactions in mechanically ventilated patients. ${ }^{26}$

The afterload of RV is mainly composed of pulmonary blood flow resistance and vascular wall tension, and right ventricular ejection is tightly coupled with the pressure and compliance of the pulmonary artery. MV-induced pulmonary artery pressure variation (changes of TP and Ppl transmitted to the pulmonary blood vessels), as well as cardiac compression caused by increased lung volume, eventually leading to right heart dysfunction. ${ }^{27}$ In addition, due to the influence of positive pressure, the size of the inferior vena cava (IVC) will also alter, which affects the venous return. As shown in online supplemental eTable 7, eve after PSM, the early TTE group had less fluid administered. Therefore, TTE may have helped directly (assessing fluid responsiveness by monitoring the RV pressure and stroke volume, as well as IVC) ${ }^{8}$ or indirectly (estimation of high LV filling pressure and diastolic dysfunction). The latter is associated with higher mortality in patients with sepsis. ${ }^{28} 29$

Recently, similar to our study, Dessap et al found that early identification of right ventricular dysfunction based on acute cor pulmonale may allow the intensivist to design an intervention that might improve acute respiratory distress syndrome patient mortality, ${ }^{30}$ and Feng et al also showed that early transthoracic echocardiography could improve the prognosis of sepsis patients by changing their management. ${ }^{3}$ These findings increase the possibility that TTE can provide physicians with useful information in the ICU. In addition, echocardiography is non-invasive and provides reliable information in most cases. Thus, although the effect of echocardiography on patient outcomes has not yet been established, experts recommend that patients undergo MV for echocardiography. They suggested that echocardiography should be performed early in the course of management to quickly obtain information on ventricular dimensions and function and to assess changes in cardiac output in response to therapy. ${ }^{31}{ }^{32}$ Our findings provide evidence for the early use of echocardiography in mechanically ventilated patients and the possibility that TTE can be better applied in the ICU.

The present analysis had some limitations. First, this was a retrospective registry study based on two large 
databases; although the multivariate regression model was used to control confounding factors, the addition of other variables may have affected the results. Besides, since much data from a retrospective study of MIMIC and eICU are not available, it is difficult to fully follow the checklist conducting and reporting critical care echocardiography (CCE) studies recommended by the Preferred Reporting Items for Critical care Echocardiography Studies (PRICES) ${ }^{33}$ which contributes to reducing the interpretation and reproducibility of CCE studies. Second, we used PSM to adjust some of the potential selection biases. Although we performed a sensitivity analysis to make the results more reliable, some biases are inevitable. Third, as this was a retrospective study, we cannot know whether the management differed between the early TTE group and the control group with regards to TTE, and there is insufficient evidence to show that different management is associated with the mortality of MV patients. Fourth, to rule out the impact of severe preexisting heart diseases or cardiac surgery, only patients from the MICU and SICU were selected. Finally, although our study suggested that the in-hospital mortality of MV patients was associated with early TTE, further prospective randomised controlled trials are still needed to validate our results.

\section{CONCLUSION}

To the best of our knowledge, this is the first report to determine the clinical value of TTE for patients undergoing MV. Early application of echocardiography during MV may be beneficial for the prognosis of patients. However, further prospective, multicentre, randomised controlled studies are needed to validate our results.

\section{Author affiliations}

${ }^{1}$ Department of Intensive Care Unit, The First Affiliated Hospital of Wenzhou Medical University, Wenzhou, Zhejiang, China

${ }^{2}$ Key Laboratory of Intelligent Treatment and Life Support for Critical Diseases of Zhejiang Provincial, Wenzhou, Zhejiang, China

${ }^{3}$ Department of Hepatobiliary and pancreatic surgery, Zhejiang University School of Medicine Second Affiliated Hospital, Hangzhou, Zhejiang, China

${ }^{4}$ Wenzhou Key Laboratory of Critical Care and Artificial Intelligence, Wenzhou, China ${ }^{5}$ Department of Blood Transfusion, The First Affiliated Hospital of Wenzhou Medical University, Wenzhou, Zhejiang, China

${ }^{6}$ The Project of Application Technology Collaborative Innovation Center of Wenzhou Institutions of Higher-Learning - Collaborative Innovation Center of Intelligence Medical Education, Wenzhou, China

Contributors XSY designed this study, collected and analysed data, and drafted the manuscript; XWZ designed this study, collected data, and drafted the manuscript; ZQC collected, compiled and analysed the data; WX and $\mathrm{HJ}$ analysed the data, interpreted the results and reviewed the manuscript; JS and WJC contributed with the study design and interpreted results; LLP and ZDF interpreted the results and reviewed the manuscript; YCY collected and compiled data; JYP designed and supervised this study and obtained funding, and is responsible for the overall content as guarantor. All authors read and approved the final manuscript.

Funding This study was supported by the National Natural Science Foundation of China (NSFC 81873949); Medical Innovation Discipline of Zhejiang Province (Critical Care Medicine, Y2015); Major project co-founded by Zhejiang Province and Ministry of Science and Technology(WKJ-ZJ-1909); Major Science and Technology Project of Wenzhou Science and Technology Bureau(2018ZY002); Wenzhou Science and
Technology Bureau (ZG2020012); The Project of Public Innovation Platform and Carrier in Zhejiang Province(2021E10016).

Competing interests None declared.

Patient consent for publication Not applicable.

Ethics approval Our study was in accordance with the ethical standards of the Declaration of Helsinki and was approved by the ethics review boards of MIT and BIDMC (researchers certification number 1605699, record id 27752407). MIMIC-III and eICU-CRD were retrospectives with lack of patient intervention, and all patients' data were de-identified; thus individual patient informed consent was not required.

Provenance and peer review Not commissioned; externally peer reviewed.

Data availability statement Data are available in a public, open access repository. Data are available upon reasonable request. All data relevant to the study are included in the article or uploaded as supplementary information. The datasets used during the current study are available from the corresponding author on reasonable request at wmupanjingye@126.com. However,reanalysis of the full data for other use requires approval by eICU-CRD (https://www.physionet.org/content/eicu-crd/2. 0/) and the MIMIC III Institute (https://mimic.mit.edu/).

Supplemental material This content has been supplied by the author(s). It has not been vetted by BMJ Publishing Group Limited (BMJ) and may not have been peer-reviewed. Any opinions or recommendations discussed are solely those of the author(s) and are not endorsed by BMJ. BMJ disclaims all liability and responsibility arising from any reliance placed on the content. Where the content includes any translated material, BMJ does not warrant the accuracy and reliability of the translations (including but not limited to local regulations, clinical guidelines, terminology, drug names and drug dosages), and is not responsible for any error and/or omissions arising from translation and adaptation or otherwise.

Open access This is an open access article distributed in accordance with the Creative Commons Attribution Non Commercial (CC BY-NC 4.0) license, which permits others to distribute, remix, adapt, build upon this work non-commercially, and license their derivative works on different terms, provided the original work is properly cited, appropriate credit is given, any changes made indicated, and the use is non-commercial. See: http://creativecommons.org/licenses/by-nc/4.0/.

\section{ORCID iDs}

Hao Jiang http://orcid.org/0000-0001-7955-1363

Wen Xu http://orcid.org/0000-0002-5311-1965

Wenjing Chen http://orcid.org/0000-0002-3167-3334

Lingling Pan http://orcid.org/0000-0001-6491-2760

Xueshu Yu http://orcid.org/0000-0003-3807-6070

Yincai Ye http://orcid.org/0000-0002-2355-559X

Zhendong Fang http://orcid.org/0000-0002-8252-8655

Xianwei Zhang http://orcid.org/0000-0003-0833-960X

Zhiqiang Chen http://orcid.org/0000-0001-7160-8345

Jie Shu http://orcid.org/0000-0003-2570-5560

Jingye Pan http://orcid.org/0000-0002-2367-1275

\section{REFERENCES}

1 Lazzeri C, Cianchi G, Bonizzoli M, et al. The potential role and limitations of echocardiography in acute respiratory distress syndrome. Ther Adv Respir Dis 2016;10:136-48.

2 Patel AR, Patel AR, Singh S, et al. Cardiac ultrasound in the intensive care unit: a review. Cureus 2019;11:e4612.

3 Feng M, McSparron JI, Kien DT, et al. Transthoracic echocardiography and mortality in sepsis: analysis of the MIMIC-III database. Intensive Care Med 2018;44:884-92.

4 Singh K, Mayo P. Critical care echocardiography and outcomes in the critically ill. Curr Opin Crit Care 2018;24:316-21.

5 American College of Cardiology Foundation Appropriate Use Criteria Task Force, American Society of Echocardiography, American Heart Association, et al. ACCF/ASE/AHA/ASNC/HFSA/HRS/SCAI/SCCM/ SCCT/SCMR 2011 appropriate use criteria for echocardiography. A report of the American College of cardiology Foundation appropriate use criteria Task force, American Society of echocardiography, American heart association, American Society of nuclear cardiology, heart failure Society of America, heart rhythm Society, Society for cardiovascular angiography and interventions, society of critical care medicine, society of cardiovascular computed tomography, and Society for cardiovascular magnetic resonance endorsed by the American College of chest physicians. J Am Coll Cardiol 2011;57:1126-66. 
6 Brun-Buisson C, Minelli C, Bertolini G. Epidemiology and outcome of acute lung injury in European intensive care units. results from the alive study. Intensive Care Med 2004;30:51-61.

7 Su L, Zhang R, Zhang Q, et al. The effect of mechanical ventilation on peripheral perfusion index and its association with the prognosis of critically ill patients. Crit Care Med 2019;47:685-90.

8 Boyd JH, Sirounis D, Maizel J, et al. Echocardiography as a guide for fluid management. Crit Care 2016;20:274.

9 Sanfilippo F, Di Falco D, Noto A, et al. Association of weaning failure from mechanical ventilation with transthoracic echocardiography parameters: a systematic review and meta-analysis. $\mathrm{Br} J$ Anaesth 2021;126:319-30.

10 Mayo P, Dessap AM, Vieillard-Baron A. Myths about critical care echocardiography: the ten false beliefs that intensivists should understand. Intensive Care Med 2015;41:1103-6.

11 von Elm E, Altman DG, Egger M, et al. The strengthening the reporting of observational studies in epidemiology (STROBE) statement: guidelines for reporting observational studies. Int J Surg 2014;12:1495-9.

12 Johnson AEW, Pollard TJ, Shen L, et al. MIMIC-III, a freely accessible critical care database. Scientific Data 2016;3:160035.

13 Pollard TJ, Johnson AEW, Raffa JD, et al. The elCU Collaborative research database, a freely available multi-center database for critical care research. Sci Data 2018;5:180178.

14 Serpa Neto A, Deliberato RO, Johnson AEW, et al. Mechanical power of ventilation is associated with mortality in critically ill patients: an analysis of patients in two observational cohorts. Intensive Care Med 2018;44:1914-22.

15 Bakbergenuly I, Hoaglin DC, Kulinskaya E. Estimation in metaanalyses of mean difference and standardized mean difference. Stat Med 2020;39:171-91.

16 Stekhoven DJ, Bühlmann P. MissForest--non-parametric missing value imputation for mixed-type data. Bioinformatics 2012;28:112-8.

17 Cole SR, Hernán MA. Constructing inverse probability weights for marginal structural models. Am J Epidemiol 2008;168:656-64.

18 Marc, Ratkovic Ket al. Covariate balancing propensity score. J R Statist Soc B 2014;76:243-63.

19 McCaffrey DF, Griffin BA, Almirall D, et al. A tutorial on propensity score estimation for multiple treatments using generalized boosted models. Stat Med 2013;32:3388-414.

20 Gattinoni L, Carlesso E, Cadringher P, et al. Physical and biological triggers of ventilator-induced lung injury and its prevention. Eur Respir J 2003;22:15s-25.
21 Magder S, Guerard B. Heart-lung interactions and pulmonary buffering: lessons from a computational modeling study. Respir Physiol Neurobiol 2012;182:60-70.

22 Jardin F, Vieillard-Baron A. Right ventricular function and positive pressure ventilation in clinical practice: from hemodynamic subsets to respirator settings. Intensive Care Med 2003;29:1426-34

23 Vieillard-Baron A, Millington SJ, Sanfilippo F, et al. A decade of progress in critical care echocardiography: a narrative review. Intensive Care Med 2019;45:770-88.

24 Vincent J-L, Rhodes A, Perel A, et al. Clinical review: Update on hemodynamic monitoring--a consensus of 16. Crit Care 2011;15:229.

25 Cecconi M, De Backer D, Antonelli M, et al. Consensus on circulatory shock and hemodynamic monitoring. Task force of the European Society of intensive care medicine. Intensive Care Med 2014:40:1795-815

26 Vignon P, Begot E, Mari A, et al. Hemodynamic assessment of patients with septic shock using Transpulmonary Thermodilution and critical care echocardiography. Chest 2018;153:55-64.

27 Pinsky MR. The right ventricle: interaction with the pulmonary circulation. Crit Care 2016;20:266.

28 Sanfilippo F, Corredor C, Fletcher N, et al. Diastolic dysfunction and mortality in septic patients: a systematic review and meta-analysis. Intensive Care Med 2015;41:1004-13.

29 Sanfilippo F, Corredor C, Arcadipane A, et al. Tissue Doppler assessment of diastolic function and relationship with mortality in critically ill septic patients: a systematic review and meta-analysis. $\mathrm{Br}$ $J$ Anaesth 2017;119:583-94.

30 Mekontso Dessap A, Boissier F, Charron C, et al. Acute cor pulmonale during protective ventilation for acute respiratory distress syndrome: prevalence, predictors, and clinical impact. Intensive Care Med 2016;42:862-70.

31 Pinsky MR, Payen D. Functional hemodynamic monitoring. Crit Care 2005;9:566-72.

32 Vieillard-Baron A, Matthay M, Teboul JL, et al. Experts' opinion on management of hemodynamics in ARDS patients: focus on the effects of mechanical ventilation. Intensive Care Med 2016;42:739-49.

33 Sanfilippo F, Huang S, Herpain A, et al. The prices statement: an ESICM expert consensus on methodology for conducting and reporting critical care echocardiography research studies. Intensive Care Med 2021;47:1-13. 